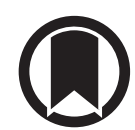

CrossMark

\title{
Confronting and mitigating the risk of COVID-19 associated pulmonary aspergillosis
}

\author{
Darius Armstrong-James ${ }^{1,13}$, Jonathan Youngs ${ }^{2}$, Tihana Bicanic ${ }^{2}$, \\ Alireza Abdolrasouli ${ }^{3}$, David W. Denning $\mathbb{1}^{4}$, Elizabeth Johnson ${ }^{5}$, Varun Mehra ${ }^{6}$, \\ Tony Pagliuca ${ }^{6}$, Brijesh Patel ${ }^{7}$, Johanna Rhodes $^{8}$, Silke Schelenz ${ }^{9}$, \\ Anand Shah $\mathbb{1}^{8,10}$, Frank L. van de Veerdonk ${ }^{11}$, Paul E. Verweij ${ }^{11}$, \\ P. Lewis White ${ }^{12}$ and Matthew C. Fisher ${ }^{8,13}$
}

\begin{abstract}
Affiliations: ${ }^{1}$ Dept of Infectious Diseases, Imperial College London, London, UK. ${ }^{2}$ Institute of Infection and Immunity, St George's University of London, London, UK. ${ }^{3}$ Dept of Medical Microbiology, North West London Pathology, Imperial College Healthcare NHS Trust, London, UK. ${ }^{4}$ Faculty of Biology, Medicine and Health, The University of Manchester, National Aspergillosis Centre, Wythenshawe Hospital and Manchester Academic Health Science Centre, Manchester, UK. ${ }^{5}$ Mycology Reference Laboratory, Public Health England National Infection Service, Bristol, UK. ${ }^{6}$ Dept of Haematological Medicine, Kings College Hospital NHS Foundation Trust, London, UK. ${ }^{7}$ Dept of Surgery and Cancer, Faculty of Medicine, Imperial College London, London, UK. ${ }^{8}$ MRC Center for Global Infectious Disease Analysis, School of Public Health, Imperial College London, London, UK. ${ }^{9}$ Infection Sciences, Kings College Hospital NHS Foundation Trust, London, UK. ${ }^{10}$ Royal Brompton and Harefield NHS Foundation Trust, London, UK. ${ }^{11}$ Dept of Medical Microbiology and Centre of Expertise in Mycology Radboudumc/CWZ, Radboud University Medical Centre, Nijmegen, The Netherlands. ${ }^{12}$ Mycology Reference Laboratory, Public Health Wales Microbiology Cardiff, Cardiff, UK. ${ }^{13}$ D. ArmstrongJames and Matthew C. Fisher contributed equally to this article as lead authors and supervised the work.
\end{abstract}

Correspondence: Darius Armstrong-James, Imperial College London, Dept of Infectious Diseases, Flowers Building, London, SW7 2AZ, UK. E-mail: d.armstrongdimperial.ac.uk

@ERSpublications

Cases of COVID-19 associated pulmonary aspergillosis (CAPA) are being increasingly reported and physicians treating patients with COVID-19-related lung disease need to actively consider these fungal co-infections https://bit.ly/3feuGsQ

Cite this article as: Armstrong-James D, Youngs J, Bicanic T, et al. Confronting and mitigating the risk of COVID-19 associated pulmonary aspergillosis. Eur Respir J 2020; 56: 2002554 [https://doi.org/10.1183/ 13993003.02554-2020].

The coronavirus disease 2019 (COVID-19) virus caused a wide spectrum of disease in healthy individuals, as well as those with common comorbidities [1]. Severe COVID-19 is characterised by acute respiratory distress syndrome (ARDS) secondary to viral pneumonitis, treatment of which may require mechanical ventilation or extracorporeal membrane oxygenation [2]. Clinicians are alert to the possibility of bacterial co-infection as a complication of lower respiratory tract viral infection; for example, a recent review found that $72 \%$ of patients with COVID-19 received antimicrobial therapy [3]. However, the risk of fungal co-infection, in particular COVID-19 associated pulmonary aspergillosis (CAPA), remains underappreciated.

Fungal disease consistent with invasive aspergillosis has been observed with other severe coronaviruses such as severe acute respiratory syndrome-coronavirus (SARS-CoV) 2003 [4, 5] and Middle East Respiratory Syndrome-coronavirus [6]. From the outset of the COVID-19 pandemic, there were warning 
signs of secondary invasive fungal infection. Aspergillus flavus was isolated from the respiratory tract in one out of 99 patients in the first COVID-19 cohort from Wuhan, China to be reported in any detail [2] and Aspergillus spp. were isolated from two (3.8\%) out of 52 patients in a subsequent cohort of critically unwell patients from this region [7]. More recently, retrospective case series from Belgium [8], France [9], The Netherlands [10] and Germany [11] have reported evidence of CAPA in an alarming 20-35\% of mechanically ventilated patients.

\section{CAPA}

Influenza-associated pulmonary aspergillosis (IAPA) presents a known risk to critically unwell patients with influenza [12-14] and the clinical course of COVID-19 shows many features that are shared with severe influenza infection. These include ARDS, lymphopenia, bilateral pulmonary infiltrates, systemic pro-inflammatory cytokine responses and sepsis leading to multiple organ failure $[14,15]$. It is therefore reasonable to suspect that patients with severe COVID-19 may be similarly susceptible to invasive aspergillosis. Corticosteroid use is an important acquired immunological risk factor for IAPA [16] and, during the SARS-CoV 2003 epidemic, there were case reports of patients developing SARS-associated invasive aspergillosis after corticosteroid use [5]. Corticosteroid use has been reported in hospitalised patients with COVID-19 [1] and may further contribute to the risk of CAPA. Importantly, the recent finding by the UK RECOVERY trial (ISRCTN50189673) [17] of a one-third mortality reduction conferred by dexamethasone in ventilated patients with COVID-19, while leading to a crucial new therapeutic avenue, may increase the risk of patients acquiring CAPA and emphasises the need for enhanced fungal surveillance.

Table 1 summarises individual patient-level data in 33 cases of CAPA that have been reported to date. The median (interquartile range) age of cases is 70 (57-75) years, of whom only two (6\%) had a European Organization for Research and Treatment of Cancer (EORTC) host factor. Of these 16 (48\%) had exposure to inhaled or systemic corticosteroids, $10(30 \%)$ had diabetes and nine $(27 \%)$ had underlying chronic lung disease; COPD $(n=5)$, asthma $(n=3)$, bullous emphysema $(n=1)$, pulmonary fibrosis $(n=1)$ and post-radiotherapy for nonsmall-cell lung cancer $(n=1)$. CAPA was diagnosed a median (interquartile range) 5.5 (4.3-9) days after intensive care unit (ICU) admission and 21 (63.6\%) patients had died by the time of publication. This mortality is in excess of most cohorts of ventilated patients with COVID-19, as a comparison in the UK ISARIC cohort $618(37 \%)$ out of 1658 ventilated patients had died by the time of publication (17\% discharged and $46 \%$ still receiving care) [23].

Invasive aspergillosis is difficult to diagnose in critically unwell patients without traditional host factors because radiological changes are usually nonspecific (e.g. infiltrates, consolidation or nodules), with features such as halo sign, air-crescent sign or cavitation being rare [24]. For these reasons SCHAUwVLIEGHE et al. [13] developed the modified AspICU criteria to help diagnose IAPA which (in the absence of histology) essentially relies on mycological evidence of Aspergillus spp. in the form of a positive bronchoalveolar lavage (BAL) culture or positive galactomannan (GM) in serum/BAL. Applying these modified AspICU criteria, five cases of CAPA in table 1 were "proven", 11 "putative" and 17 might be considered putative but with caveats which have been described in the table. For example, in many cases a tracheal aspirate, rather than BAL, provided the only mycological evidence of invasive aspergillosis (in the absence of tracheobronchitis/cavitation). There should therefore be caution about over-estimating the incidence of CAPA from such case series, which may include some patients with aspergillus colonisation or contamination only. In the study by ALANIO et al. [9] which reported evidence of CAPA in nine (33\%) out of 27 ventilated patients who underwent BAL/tracheal aspirate, one case was defined based on a BAL GM of 0.89 (below the usual cut-off of 1.0), two based on tracheal aspirate rather than BAL culture, one based on a serum GM of 0.51 (cut-off being 0.50 ) and in four cases BAL culture was positive but BAL GM negative, which suggests a lack of tissue invasion. Indeed, out of seven cases that were not treated with antifungals, five survived. Accordingly, larger, prospective, multi-site studies are needed to refine the AspICU criteria for patients with COVID-19, as well as to estimate incidence and the impact of CAPA on survival $[25,26]$.

\section{Diagnosis and risk of CAPA}

Bearing these observations in mind, we argue that critically ill patients with COVID-19 and progressive features should be screened for CAPA. We acknowledge that acquiring and handling clinical samples for microbiology is very challenging given the Hazard Group 3 rating of the SARS-CoV-2 virus, alongside an overburdened critical care service [27].

Ideally, screening for CAPA entails using a combination of computed tomography chest imaging and Aspergillus antigen tests on BAL and serum including GM ELISA or lateral-flow tests [28], or aspergillus PCR [29]. Whilst characteristic CT features of invasive aspergillosis such as nodules with halo sign were 


\begin{tabular}{|c|c|c|c|c|c|c|c|c|c|c|c|c|c|c|}
\hline Location [ref.] & $\begin{array}{l}\text { Age } \\
\text { years }\end{array}$ & Sex & IPA risk factors & Radiology & BAL culture & TA culture & BAL GM & $\begin{array}{c}\text { Serum } \\
\text { GM }\end{array}$ & $\begin{array}{c}\text { Other } \\
\text { diagnostics }\end{array}$ & $\begin{array}{c}\text { Onset } \\
\text { days } \\
\text { post-ICU }\end{array}$ & EORTC status & $\begin{array}{l}\text { Mod } \\
\text { AspICU } \\
\text { status }\end{array}$ & Treatment & Outcome \\
\hline $\begin{array}{l}\text { Cologne, } \\
\text { Germany [11] }\end{array}$ & 62 & $\mathrm{~F}$ & $\begin{array}{l}\text { Ex-smoker, moderate } \\
\text { COPD, inhaled steroids }\end{array}$ & $\begin{array}{l}\text { Ground-glass } \\
\text { opacities, } \\
\text { crazy paving, } \\
\text { peripheral } \\
\text { nodular } \\
\text { consolidation }\end{array}$ & A. fumigatus & $N R$ & $(+)>2.5$ & $(-)$ & $\begin{array}{l}\text { BAL PCR } \\
\text { A. fumigatus }\end{array}$ & NR & No host factor ${ }^{\#}$ & Putative & v & Died \\
\hline $\begin{array}{l}\text { Cologne, } \\
\text { Germany [11] }\end{array}$ & 70 & M & Ex-smoker & $\begin{array}{c}\text { Ground-glass } \\
\text { opacities, } \\
\text { occasional } \\
\text { nodules }\end{array}$ & $(-)$ & $N R$ & $(+)>2.5$ & l+ 0.7 & $\begin{array}{l}\text { BAL PCR } \\
\text { A. fumigatus }\end{array}$ & NR & No host factor & Putative & 1 & Died \\
\hline $\begin{array}{l}\text { Cologne, } \\
\text { Germany [11] }\end{array}$ & 54 & M & $\begin{array}{c}\text { Diabetes, systemic } \\
\text { corticosteroids } \\
0.4 \mathrm{mg} \cdot \mathrm{kg}^{-1} \cdot \mathrm{day}^{-1} \times 13 \text { days }\end{array}$ & $\begin{array}{l}\text { Ground-glass } \\
\text { opacities, } \\
\text { nodular } \\
\text { infiltrates with } \\
\text { cavities, air } \\
\text { crescent sign }\end{array}$ & $(-)$ & A. fumigatus & $(+)>2.5$ & $(-)$ & $\begin{array}{l}\text { BAL PCR } \\
\text { A. fumigatus }\end{array}$ & $N R$ & No host factor & Putative & $\mathrm{C}, \mathrm{V}$ & Alive \\
\hline $\begin{array}{l}\text { Cologne, } \\
\text { Germany [11] }\end{array}$ & 73 & $M$ & $\begin{array}{l}\text { Smoker, bullous } \\
\text { emphysema, severe COPD, } \\
\text { inhaled steroids }\end{array}$ & $\begin{array}{l}\text { Ground-glass } \\
\text { opacities, } \\
\text { occasional } \\
\text { nodules, } \\
\text { known bullous } \\
\text { emphysema }\end{array}$ & ND & A. fumigatus & ND & $(-)$ & $\begin{array}{l}\text { TA PCR } \\
\text { A. fumigatus }\end{array}$ & $N R$ & No host factor & $\begin{array}{l}\text { Putative } \\
\text { only if TA } \\
\text { considered } \\
\text { equivalent } \\
\text { to BAL }\end{array}$ & V & Died \\
\hline $\begin{array}{l}\text { Cologne, } \\
\text { Germany [11] }\end{array}$ & 54 & $\mathrm{~F}$ & None & $\begin{array}{l}\text { Ground-glass } \\
\text { opacities, } \\
\text { crazy paving, } \\
\text { central and } \\
\text { peripheral } \\
\text { consolidation, } \\
\text { smaller } \\
\text { nodular } \\
\text { infiltrates }\end{array}$ & ND & $(-)$ & ND & $\begin{array}{l}(+) 2.7 \\
1.3\end{array}$ & TA PCR (-) & NR & No host factor & Putative & C, V & Alive \\
\hline $\begin{array}{l}\text { Munich, } \\
\text { Germany [18] }\end{array}$ & 80 & M & Pulmonary fibrosis & $\begin{array}{l}\text { Typical signs } \\
\text { for COVID-19 } \\
\text { pneumonia } \\
\text { but no } \\
\text { specific signs } \\
\text { for IPA }\end{array}$ & A. fumigatus & $N R$ & $(+)>6$ & (+) 1.5 & & 5 & No host factor & Putative & L-AmB & Died \\
\hline $\begin{array}{l}\text { Munich, } \\
\text { Germany [18] }\end{array}$ & 70 & M & None & $\begin{array}{l}\text { Typical signs } \\
\text { for COVID-19 } \\
\text { pneumonia } \\
\text { but no } \\
\text { specific signs } \\
\text { for IPA }\end{array}$ & A. fumigatus & NR & $(+)>6$ & $(-)$ & & 6 & No host factor & Putative & L-AmB & Died \\
\hline
\end{tabular}




\begin{tabular}{|c|c|c|c|c|c|c|c|c|c|c|c|c|c|c|}
\hline Location [ref.] & $\begin{array}{l}\text { Age } \\
\text { years }\end{array}$ & Sex & IPA risk factors & Radiology & BAL culture & TA culture & BAL GM & $\begin{array}{c}\text { Serum } \\
\text { GM }\end{array}$ & $\begin{array}{c}\text { Other } \\
\text { diagnostics }\end{array}$ & $\begin{array}{c}\text { Onset } \\
\text { days } \\
\text { post-ICU }\end{array}$ & EORTC status & $\begin{array}{l}\text { Mod } \\
\text { AspICU } \\
\text { status }\end{array}$ & Treatment & t Outcome \\
\hline $\begin{array}{l}\text { Paris, France } \\
\text { [19] }\end{array}$ & 74 & M & Myelodysplastic syndrome & NR & ND & A. fumigatus & ND & $(-) \times 2$ & $\begin{array}{c}\text { TA PCR } \\
\text { A. fumigatus } \times 2, \\
\text { TA GM (-) } \times 1, \text { BDG } \\
\text { and serum PCR } \\
\text { (-) } \times 2\end{array}$ & 4 & No host factor & $\begin{array}{l}\text { Putative } \\
\text { only if TA } \\
\text { considered } \\
\text { equivalent } \\
\text { to BAL }\end{array}$ & None & Died \\
\hline Paris, France [9] & 53 & M & $\begin{array}{c}\text { Dexamethasone } \\
20 \mathrm{mg}^{\text {day }}{ }^{-1} \text { days } 1-5 \\
10 \mathrm{mg} \cdot \text { day }^{-1} \text { days } 6-10\end{array}$ & $\begin{array}{c}\text { Typical } \\
\text { COVID-19 }\end{array}$ & $(-)$ & NR & $(-) 0.89$ & $(-)$ & $\begin{array}{l}\text { BAL PCR }(-), \\
\text { Serum PCR }(-), \\
\text { BDG }(+)>500\end{array}$ & NR & No host factor & $\begin{array}{l}\text { Putative } \\
\text { only if BAL } \\
\text { GM cut-off } \\
\text { lowered to } \\
>0.8\end{array}$ & None & Alive \\
\hline Paris, France [9] & 59 & $\mathrm{~F}$ & Diabetes & $\begin{array}{c}\text { Typical } \\
\text { COVID-19 }\end{array}$ & A. fumigatus & NR & $(-)$ & $(-)$ & $\begin{array}{l}\text { BAL PCR (-), } \\
\text { serum PCR (-) }\end{array}$ & NR & No host factor & $\begin{array}{c}\text { Putative } \\
\text { but note } \\
\text { BAL } \\
\text { culture (+) } \\
\text { but BAL } \\
\text { GM (-) }\end{array}$ & None & Alive \\
\hline Paris, France [9] & 69 & $\mathrm{~F}$ & 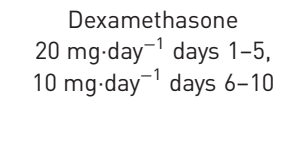 & $\begin{array}{c}\text { Typical } \\
\text { COVID-19 }\end{array}$ & ND & A. fumigatus & ND & $(-)$ & $\begin{array}{c}\text { TA PCR } \\
\text { A. fumigatus, } \\
\text { serum PCR (-), } \\
\text { BDG (-) }\end{array}$ & NR & No host factor & $\begin{array}{l}\text { Putative } \\
\text { only if TA } \\
\text { considered } \\
\text { equivalent } \\
\text { to BAL }\end{array}$ & None & Alive \\
\hline Paris, France [9] & 63 & $\mathrm{~F}$ & $\begin{array}{l}\text { Diabetes, dexamethasone } \\
20 \mathrm{mg}^{- \text {day }^{-1}} \text { days } 1-5 \\
10 \mathrm{mg}^{- \text {day }^{-1} \text { days } 6-10}\end{array}$ & $\begin{array}{c}\text { Typical } \\
\text { COVID-19 }\end{array}$ & $(-)$ & NR & $(-)$ & $(+) 0.51$ & $\begin{array}{l}\text { BAL PCR (-), } \\
\text { BDG }(+) 105\end{array}$ & NR & No host factor & $\begin{array}{c}\text { Putative } \\
\text { but relies } \\
\text { on serum } \\
\text { GM of only } \\
0.51\end{array}$ & None & Died \\
\hline Paris, France [9] & 43 & M & Asthma, corticosteroids & $\begin{array}{c}\text { Typical } \\
\text { COVID-19 }\end{array}$ & A. fumigatus & NR & $(-)$ & $(-)$ & $\begin{array}{l}\text { BAL PCR (-), } \\
\text { serum PCR (-), } \\
\text { BDG (-) }\end{array}$ & NR & No host factor & $\begin{array}{c}\text { Putative } \\
\text { but note } \\
\text { BAL } \\
\text { culture (+) } \\
\text { but BAL } \\
\text { GM (-) }\end{array}$ & None & Alive \\
\hline Paris, France [9] & 79 & M & $\begin{array}{l}\text { Diabetes, Dexamethasone } \\
20 \mathrm{mg} \cdot \mathrm{d}^{-1} \text { days } 1-5 \\
10 \mathrm{mg} \cdot \mathrm{d}^{-1} \text { days } 6-10\end{array}$ & $\begin{array}{c}\text { Typical } \\
\text { CoVID-19, } \\
\text { segmental } \\
\text { lung } \\
\text { atelectasis }\end{array}$ & A. fumigatus & NR & $(-)$ & $(-)$ & $\begin{array}{c}\text { BAL PCR } \\
\text { A. fumigatus, } \\
\text { serum PCR (-), } \\
\text { BDG (-) }\end{array}$ & NR & No host factor & $\begin{array}{c}\text { Putative } \\
\text { but note } \\
\text { BAL } \\
\text { culture (+) } \\
\text { but BAL } \\
\text { GM (-) }\end{array}$ & None & Alive \\
\hline Paris, France [9] & 77 & M & $\begin{array}{l}\text { Asthma, dexamethasone } \\
20 \mathrm{mg} \cdot \text { day }^{-1} \text { days } 1-5, \\
10 \mathrm{mg} \cdot \text { day }^{-1} \text { days } 6-10\end{array}$ & $\begin{array}{l}\text { "Typical } \\
\text { CoVID-19", } \\
\text { emphysema }\end{array}$ & A. fumigatus & NR & $(+) 3.9$ & $(-)$ & $\begin{array}{c}\text { BAL PCR } \\
\text { A. fumigatus, } \\
\text { serum PCR (-), } \\
\text { BDG (+) } 135\end{array}$ & NR & No host factor & Putative & $\mathrm{v}$ & Died \\
\hline Paris, France [9] & 75 & $\mathrm{~F}$ & $\begin{array}{l}\text { Diabetes, dexamethasone } \\
20 \mathrm{mg}^{\text {day }}{ }^{-1} \text { days } 1-5 \\
10 \mathrm{mg} \cdot \text { day }^{-1} \text { days } 6-10\end{array}$ & $\begin{array}{c}\text { Typical } \\
\text { COVID-19 }\end{array}$ & A. fumigatus & NR & $(-)$ & $(-)$ & $\begin{array}{l}\text { BAL PCR, } \\
\text { A. fumigatus, } \\
\text { serum PCR (-), } \\
\text { BDG (+) } 450\end{array}$ & NR & No host factor & $\begin{array}{c}\text { Putative } \\
\text { but note } \\
\text { BAL } \\
\text { culture (+) } \\
\text { but BAL } \\
\text { GM (-) }\end{array}$ & c & Died \\
\hline
\end{tabular}




\begin{tabular}{|c|c|c|c|c|c|c|c|c|c|c|c|c|c|c|}
\hline Location [ref.] & $\begin{array}{c}\text { Age } \\
\text { years }\end{array}$ & Sex & IPA risk factors & Radiology & BAL culture & TA culture & BAL GM & $\begin{array}{l}\text { Serum } \\
\text { GM }\end{array}$ & $\begin{array}{c}\text { Other } \\
\text { diagnostics }\end{array}$ & $\begin{array}{c}\text { Onset } \\
\text { days } \\
\text { post-ICU }\end{array}$ & EORTC status & $\begin{array}{l}\text { Mod } \\
\text { AspICU } \\
\text { status }\end{array}$ & Treatment & Outcome \\
\hline Paris, France [9] & 47 & M & Myeloma, corticosteroids & $\begin{array}{c}\text { Typical } \\
\text { CoVID-19, one } \\
\text { peripheral } \\
\text { nodule }\end{array}$ & ND & A. fumigatus & ND & $(-)$ & $\begin{array}{c}\text { TA PCR } \\
\text { A. fumigatus, } \\
\text { serum PCR (-), } \\
\operatorname{BDG~(-)~}\end{array}$ & $N R$ & Probable & $\begin{array}{c}\text { Putative } \\
\text { only if TA } \\
\text { considered } \\
\text { equivalent } \\
\text { to BAL }\end{array}$ & None & Died \\
\hline $\begin{array}{l}\text { Graz, Austria } \\
\text { [20] }\end{array}$ & 70 & M & $\begin{array}{l}\text { Moderate COPD, steroid } \\
\text { inhaler, obstructive sleep } \\
\text { apnoea, diabetes }\end{array}$ & $\begin{array}{l}\text { Ground-glass } \\
\text { opacities, } \\
\text { crazy paving, } \\
\text { reversed halo } \\
\text { sign, } \\
\text { progression of } \\
\text { the bilateral } \\
\text { infiltrates on } \\
\text { day } 2 \text { chest } \\
\text { radiography }\end{array}$ & ND & A. fumigatus & ND & $(-)$ & $\begin{array}{c}\text { TA LFD (+), BDG } \\
(-)\end{array}$ & 3 & No host factor & $\begin{array}{c}\text { Putative } \\
\text { only if TA } \\
\text { considered } \\
\text { equivalent } \\
\text { to BAL }\end{array}$ & $\mathrm{v}$ & Died \\
\hline $\begin{array}{l}\text { Antwerp, } \\
\text { Belgium [8] }\end{array}$ & 86 & M & None & ND & ND & A. flavus & ND & $(-)$ & & 9 & No host factor & $\begin{array}{l}\text { Putative } \\
\text { only if TA } \\
\text { considered } \\
\text { equivalent } \\
\text { to BAL }\end{array}$ & None & Died \\
\hline $\begin{array}{l}\text { Antwerp, } \\
\text { Belgium [8] }\end{array}$ & 38 & M & None & $(+)$ & A. fumigatus & $N R$ & $(+)>2.8$ & $(-)$ & $\begin{array}{l}\text { Histology from } \\
\text { bronchoscopy (+) }\end{array}$ & 6 & Proven & Proven & $V_{1} I$ & Alive \\
\hline $\begin{array}{l}\text { Antwerp, } \\
\text { Belgium [8] }\end{array}$ & 62 & M & Diabetes & ND & A. fumigatus & NR & $(+)>2.0$ & $(-)$ & $\begin{array}{l}\text { Histology from } \\
\text { bronchoscopy (+) }\end{array}$ & 16 & Proven & Proven & v & Died \\
\hline $\begin{array}{l}\text { Antwerp, } \\
\text { Belgium [8] }\end{array}$ & 73 & M & Diabetes & ND & A. fumigatus & NR & $(+)>2.8$ & $(-)$ & $\begin{array}{l}\text { Histology from } \\
\text { bronchoscopy (+) }\end{array}$ & 5 & Proven & Proven & V & Alive \\
\hline $\begin{array}{l}\text { Antwerp, } \\
\text { Belgium [8] }\end{array}$ & 77 & M & $\begin{array}{l}\text { Diabetes, chronic } \\
\text { corticosteroids for } \\
\text { pemphigus foliaceous }\end{array}$ & ND & A. fumigatus & NR & (+) 2.79 & $(-)$ & $\begin{array}{l}\text { Histology from } \\
\text { bronchoscopy (+) }\end{array}$ & 2 & Proven & Proven & V & Alive \\
\hline $\begin{array}{l}\text { Antwerp, } \\
\text { Belgium [8] }\end{array}$ & 55 & M & $\begin{array}{l}\text { HIV (CD4 count >250, viral } \\
\text { load <20) copies) }\end{array}$ & ND & $(-)$ & NR & $(-)$ & $(+) 0.8$ & $\begin{array}{l}\text { Histology from } \\
\text { bronchoscopy (-) }\end{array}$ & 13 & No host factor & $\begin{array}{c}\text { Putative } \\
\text { but relies } \\
\text { on serum } \\
\text { GM of only } \\
0.8\end{array}$ & $V_{1} I$ & Died \\
\hline $\begin{array}{l}\text { Antwerp, } \\
\text { Belgium [8] }\end{array}$ & 75 & M & AML with IPA 2012 & ND & A. fumigatus & NR & $(+) 2.63$ & ND & & 8 & No host factor & Putative & V & Died \\
\hline $\begin{array}{l}\text { Breda, The } \\
\text { Netherlands } \\
\text { [10] }\end{array}$ & 83 & M & $\begin{array}{c}\text { Prednisolone } \\
0.13 \mathrm{mg} \cdot \mathrm{kg}^{-1} \cdot \text { day }^{-1} \times 28 \text { days } \\
\text { for cardiomyopathy }\end{array}$ & NR & ND & A. fumigatus & ND & $(-)$ & & 3 & $\begin{array}{l}\text { Probable if steroid } \\
\text { requirement } \\
\text { reduced to } \\
<0.3 \mathrm{mg} \cdot \mathrm{kg}^{-1} \cdot \mathrm{day}^{-1}\end{array}$ & $\begin{array}{c}\text { Putative } \\
\text { only if TA } \\
\text { considered } \\
\text { equivalent } \\
\text { to BAL }\end{array}$ & $\begin{array}{l}V+A \text {, or } \\
L-A m B\end{array}$ & Died \\
\hline $\begin{array}{l}\text { Breda, The } \\
\text { Netherlands } \\
\text { [10] }\end{array}$ & 67 & M & $\begin{array}{l}\text { Severe COPD, Post RT for } \\
\text { NSCLC 2014, prednisolone } \\
0.37 \mathrm{mg} \cdot \mathrm{kg}^{-1} \cdot \text { day }^{-1} \times 2 \text { days }\end{array}$ & NR & ND & A. fumigatus & ND & ND & & 3 & No host factor & $\begin{array}{l}\text { Putative } \\
\text { only if TA } \\
\text { considered } \\
\text { equivalent } \\
\text { to BAL }\end{array}$ & $\begin{array}{l}V+A \text {, or } \\
L-A m B\end{array}$ & Died \\
\hline
\end{tabular}




\begin{tabular}{|c|c|c|c|c|c|c|c|c|c|c|c|c|c|c|}
\hline Location [ref.] & $\begin{array}{l}\text { Age } \\
\text { years }\end{array}$ & Sex & IPA risk factors & Radiology & BAL culture & TA culture & BAL GM & $\begin{array}{c}\text { Serum } \\
\text { GM }\end{array}$ & $\begin{array}{c}\text { Other } \\
\text { diagnostics }\end{array}$ & $\begin{array}{c}\text { Onset } \\
\text { days } \\
\text { post-ICU }\end{array}$ & EORTC status & $\begin{array}{l}\text { Mod } \\
\text { AspICU } \\
\text { status }\end{array}$ & Treatment & Outcome \\
\hline $\begin{array}{l}\text { Breda, The } \\
\text { Netherlands } \\
\text { [10] }\end{array}$ & 75 & M & Moderate COPD & NR & A. fumigatus & NR & $(+) 4.0$ & ND & $\begin{array}{l}\text { Mucoid white } \\
\text { sputum left } \\
\text { bronchus at } \\
\text { bronchoscopy }\end{array}$ & 5 & No host factor & Putative & $\begin{array}{l}V+A \text {, or } \\
L-A m B\end{array}$ & Died \\
\hline $\begin{array}{l}\text { Breda, The } \\
\text { Netherlands } \\
\text { [10] }\end{array}$ & 43 & M & None & NR & $(-)$ & NR & $1+13.8$ & $(-)$ & & 14 & No host factor & Putative & $\begin{array}{l}V+A \text {, or } \\
L-A m B\end{array}$ & Alive \\
\hline $\begin{array}{l}\text { Breda, The } \\
\text { Netherlands } \\
\text { [10] }\end{array}$ & 57 & M & Asthma, inhaled steroids & NR & A. fumigatus & NR & $(+) 1.6$ & $(-)$ & & 5 & No host factor & Putative & $\begin{array}{l}\text { V+A, or } \\
L-A m B\end{array}$ & Died \\
\hline $\begin{array}{l}\text { Breda, The } \\
\text { Netherlands } \\
\text { [10] }\end{array}$ & 58 & M & None & NR & ND & A. fumigatus & ND & ND & & 28 & No host factor & $\begin{array}{l}\text { Putative } \\
\text { only if TA } \\
\text { considered } \\
\text { equivalent } \\
\text { to BAL }\end{array}$ & $\begin{array}{l}V+A \text {, or } \\
L-A m B\end{array}$ & Alive \\
\hline $\begin{array}{l}\text { Paris, France } \\
\text { [21] }\end{array}$ & 80 & M & None & $\begin{array}{c}\text { Pleural } \\
\text { effusions, } \\
\text { alveolar } \\
\text { condensation, } \\
\text { ground-glass } \\
\text { opacities, } \\
\text { pulmonary } \\
\text { cysts }\end{array}$ & ND & A. flavus & ND & ND & & NR & No host factor & $\begin{array}{l}\text { Putative } \\
\text { only if TA } \\
\text { considered } \\
\text { equivalent } \\
\text { to BAL }\end{array}$ & V, I & Died \\
\hline Milan, Italy [22] & 73 & M & Diabetes & $\begin{array}{l}\text { Interstitial } \\
\text { opacities with } \\
\text { right upper } \\
\text { lobe focal } \\
\text { consolidation } \\
\text { which } \\
\text { progressively } \\
\text { worsened }\end{array}$ & A. fumigatus & NR & ND & $1+18.6$ & $\begin{array}{c}\text { Lung histology } \\
\text { from PM (+), PM } \\
\text { tissue PCR } \\
\text { Aspergillus spp. }\end{array}$ & 9 & Proven & Proven & L-AmB & Died \\
\hline Summary & $\begin{array}{c}\text { Median } \\
\text { (IQR) } \\
70 \text { (57- } \\
75)\end{array}$ & $\begin{array}{l}\text { M: } 26 \\
\text { (79\%) } \\
\text { out of } \\
33\end{array}$ & $\begin{array}{l}\text { EORTC host factor: } \mathrm{n}=2 \\
\text { (6\%); inhaled/systemic } \\
\text { steroid exposure: } \mathrm{n}=16 \\
(48 \%) \text {; diabetes: } \mathrm{n}=10 \\
(30 \%) \text {; chronic lung } \\
\text { disease: } \mathrm{n}=9(27 \%)\end{array}$ & $\begin{array}{c}\text { Nodules: } \mathrm{n}=6 \\
\text { (31.6\%), } \\
\text { cavity/ } \\
\text { halo-sign: } \mathrm{n}=2 \\
(10.5 \%)\end{array}$ & $\begin{array}{l}16(72.7 \%) \\
\text { out of } 22 \text { with } \\
\text { BAL }\end{array}$ & & $\begin{array}{c}14 \\
\text { (66.7\%) } \\
\text { out of } \\
21 \text { with } \\
\text { BAL GM }\end{array}$ & $\begin{array}{c}6 \\
\text { (21.4\%) } \\
\text { out of } \\
28 \text { with } \\
\text { serum } \\
\text { GM }\end{array}$ & & $\begin{array}{c}\text { Median } \\
\text { (IQR) } \\
5.5 \text { (4.3- } \\
\text { 9) }\end{array}$ & $\begin{array}{c}\text { Proven: } n=5 ; \\
\text { probable: } n=1 ; n o \\
\text { host factor: } n=27\end{array}$ & $\begin{array}{l}\text { Proven: } \\
\text { n=5; } \\
\text { putative: } \\
\mathrm{n}=11 ; \\
\text { putative } \\
\text { with } \\
\text { caveats: } \\
\mathrm{n}=17\end{array}$ & $\begin{array}{c}24(72.7 \%) \\
\text { treated }\end{array}$ & $\begin{array}{c}21 \\
(63.6 \%) \\
\text { died }\end{array}$ \\
\hline
\end{tabular}

IPA: invasive pulmonary aspergillosis; BAL: bronchoalveolar lavage; TA: tracheal aspirate; GM: galactomannan; EORTC: European Organization for Research and Treatment of Cancer; AspICU: clinical criteria to diagnose IPA; M: male; F: female; BDG: (1-3)- $\beta$-D-glucan; LFD: Aspergillus lateral-flow device; PM: post mortem; AML: acute myeloid leukaemia; RT: radiotherapy; NSCLC: nonsmall-cell lung cancer; NR: not recorded; V: voriconazole; I: isavuconazole; L-AmB: liposomal amphotericin B; C: caspofungin; A: anidulafungin; ND: no data; $(+)$ : positive result; (-): negative result. " : without histological evidence of "proven" IPA a patient host factor (e.g. recent neutropenia, haematological malignancy) is required to meet the probable/possible definition, corticosteroids must be given at $\geqslant 0.3 \mathrm{mg} \cdot \mathrm{kg}^{-1}$ for $\geqslant 3$ weeks to classify as a host factor result. 
seen in $17.6 \%$ of severely ill COVID-19 patients, they were not confirmed to be IPA [30]. Given the lack of typical invasive aspergillosis features on CT in IAPA, the absence of classical findings such as cavitation should not be used to exclude CAPA; however, their presence can help support the diagnosis and reduce the burden of evidence placed on mycological investigations.

In a study of 26 ICU patients that were diagnosed with proven (non-CAPA/IAPA) IPA post mortem, serum GM had only $25 \%$ sensitivity in those that were not neutropenic (versus $70 \%$ in neutropenic patients) [31]. In contrast, BAL GM was $88-90 \%$ sensitive in both groups. In the IAPA study by SchAuWvLIEGHE et al. [13] serum GM testing performed better with 20 (65\%) out of 31 positive cases, however BAL GM remained superior at $67(88 \%)$ out of 76 cases. In CAPA cases reported to date (table 1), BAL culture and GM had a sensitivity of $72.7 \%$ and $66.7 \%$, respectively, but serum GM was positive in only six (21.4\%) out of 28. Moreover, of the five cases of proven CAPA reported to date, four were serum GM negative (table 1) [8], indicating that serum GM test performance might be inferior in diagnosing CAPA. Therefore, bronchoscopy, including tracheobronchial inspection and BAL sampling for culture and GM should be the diagnostic gold standards whenever CAPA is suspected, providing this is compatible with local infection prevention and control guidance for aerosol-generating procedures. A positive BAL GM (index >1.0) would be indicative of CAPA, whereas if the index is $<0.5$ CAPA is much less likely [31]. A positive serum GM result $(\geqslant 0.5)$ would be highly suspicious for CAPA but a negative result should not be used to exclude the diagnosis. Novel lateral-flow antigen tests may represent a locally implementable alternative to GM ELISA in the CL3 laboratory, but currently require validation in ICU patients without EORTC host factors including COVID-19 [28]. An Aspergillus-specific PCR test [29] may also be helpful and if positive could also lead to the application of molecular testing for the recognised markers of clinically or environmentally derived azole resistance [32].

\section{Trigger:}

Microbiological: sputum/TA aspergillus culture positive Clinical: deteriorating or persistent poor respiratory function with no other explanation, or progressive radiology
Considerations:

If BAL not possible, consider NBL

BDG may be more sensitive than serum GM

BDG is not specific for IA, also consider candidiasis or PCP

Very high BAL GM index (>3) suggestive of CAPA

Biomarker test combinations may be superior compared to single tests

Serum biomarker negativity in the absence of BAL testing does not exclude IA
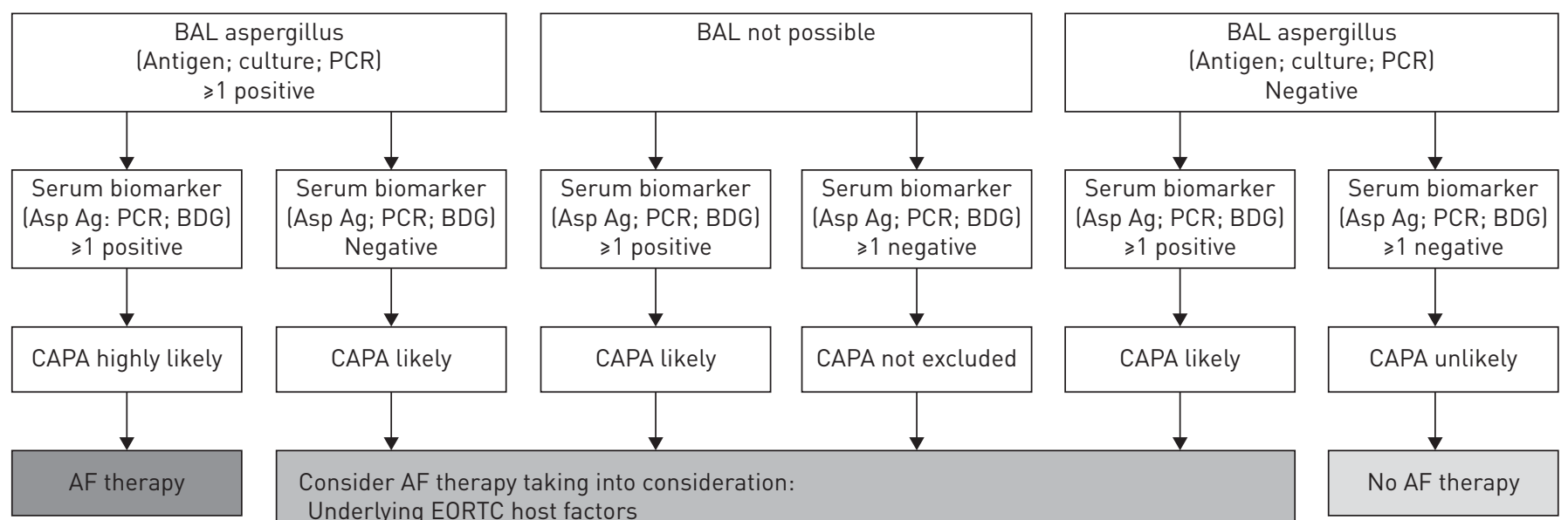

Consider AF therapy taking into consideration:

Underlying EORTC host factors

Risk factors (co-infection with influenza, corticosteroids/other immunomodulators)

Clinical deterioration with no other explanation

New nodules with cavitation or halo sign or consolidation

Follow-up serum biomarker results

Progressive pulmonary infiltrates

FIGURE 1 Proposed screening and diagnostic algorithm for coronavirus disease-2019 associated pulmonary aspergillosis (CAPA). BAL: bronchoalveolar lavage; BDG: (1-3)-3-D-glucan; TA: tracheal aspirate; Asp Ag: aspergillus antigen; AF: antifungal; IA: invasive aspergillosis; GM: galactomannan; EORTC: European Organization for Research and Treatment of Cancer; NBL: non-directed bronchoalveolar lavage; PCP: Pneumocystis pneumonia. 
A (1-3)- $\beta$-D-glucan (BDG) test on a serum sample is an easily obtained, early screening test when there is a suspicion of IPA. Although performance might be superior to serum Aspergillus antigen testing for the detection of IPA in the ICU [33], BDG negativity cannot be used to rule out infection, with a $77 \%$ sensitivity determined across a heterogeneous population of invasive aspergillosis patients, and performance in CAPA as yet to be determined. BDG positivity can occur due to a number of reasons in this patient cohort, however serial positive tests increases specificity and should prompt a diagnostic work-up including computed tomography and bronchoscopy and testing for Aspergillus antigen as outlined above [34]. While initiating antifungal treatment pre-emptively based on BDG positivity may be an improvement on empirical therapy, every effort should be made to utilise other more specific diagnostic tests to complement the BDG result.

Current guidelines advise against routine diagnostic bronchoscopy due to the risk of aerosol generation; recommending it only in patients in whom nasopharyngeal cultures are negative and BAL sampling will change clinical management [35]. In practice many patients with suspected CAPA undergo endotracheal sampling or non-directed BAL sampling only, and it is important that any case definition proposed for CAPA reflects this reality. To acknowledge this, we propose a screening and diagnostic algorithm for CAPA, which has clinical (respiratory) deterioration and/or positive aspergillus sputum, or tracheal aspirate culture as its entry point (figure 1). Although the host risk factors and clinical characteristics of CAPA are not yet understood, those individuals fulfilling the criteria for proven or probable aspergillosis $[13,14]$ should then be treated according to current guidelines [36, 37]. Importantly, now that adjunctive use of dexamethasone is likely to become widespread in the treatment of patients with severe COVID-19 [17], intensified screening for invasive aspergillosis is indicated to study the possible association between corticosteroid usage and CAPA.

Finally, the use of immunomodulatory drugs such as anakinra (recombinant interleukin-1Ra), tocilizumab (anti-interleukin-6) and Janus kinase inhibitors, currently undergoing trials for COVID-19, may also predispose patients to CAPA. There is also an increased risk of Aspergillus exposure for patients who are treated in hospital wards or makeshift "hospital" facilities that do not meet ICU specifications for appropriate room ventilation and air changes. It is also worth bearing in mind that pulmonary aspergillosis could develop into a chronic cavitary disease in a subset of patients, perhaps in those developing post-COVID-19 pulmonary fibrosis. For these reasons, clinicians following up patients manifesting chronic respiratory problems following their primary COVID-19 infection should bear in mind longer term fungal complications.

\section{Conclusion}

Fungal infections present an additional threat in the challenging task of managing COVID-19 patients in outbreak conditions. The pandemic of SARS-CoV-2 virus will undoubtedly involve CAPA, and the use of immunomodulatory therapy and impact of overburdened critical care services during this pandemic may exaggerate its impact. More research is needed on the epidemiology and diagnosis of CAPA in patients with COVID-19, a need that is partially met as ongoing prospective multi-site clinical studies are extended to include this cohort (e.g. AspiFlu) [25] or are launched (CAPA) [26] in response to the COVID-19 pandemic.

Conflict of interest: D. Armstrong-James reports grants and personal fees from Pulmocide Ltd and Gilead Sciences, outside the submitted work. J. Youngs reports funding from the UK and Ireland Gilead Fellowship Programme. T. Bicanic reports grants and personal fees for lectures from Gilead Sciences Ltd, personal fees for lectures from Pfizer, outside the submitted work. A. Abdolrasouli has received speaker honoraria from Gilead Sciences Ltd, outside the submitted work. D.W. Denning and family hold founder shares in F2G Ltd, a University of Manchester spin-out antifungal discovery company; D.W. Denning acts or has recently acted as a consultant to Scynexis, Pulmatrix, Pulmocide, Zambon, iCo Therapeutics, Mayne Pharma, Biosergen, and Fujifilm; and has been paid for talks on behalf of Hikma, Gilead, Merck, Mylan and Pfizer; D.W. Denning is a longstanding member of the Infectious Disease Society of America Aspergillosis Guidelines group, the European Society for Clinical Microbiology and Infectious Diseases Aspergillosis Guidelines group. E. Johnson has nothing to disclose. V. Mehra reports personal fees for lectures from Pfizer, Novartis, Incyte and Gilead Sciences, grants from MSD, outside the submitted work. T. Pagliuca reports personal fees for advisory board work from Gilead, MSD and Pfizer, outside the submitted work. B. Patel has nothing to disclose. J. Rhodes has nothing to disclose. S. Schelenz reports personal fees for lectures from Gilead Sciences and Pfizer, outside the submitted work. A. Shah reports grant funding from Gilead Sciences and Vertex Pharmaceuticals, and speaker fees from Gilead Sciences and Pfizer. F.L. van de Veerdonk has nothing to disclose. P.E. Verweij has received research grants from Gilead Sciences, F2G Ltd, Pfizer, Thermofisher and Merck, has served on an advisory board for Gilead Sciences, Mundipharma and F2G, and has received speaker honoraria from F2G, Mundipharma, Pfizer and Gilead Sciences. P.L. White reports personal fees from Gilead, Pfizer and F2G, grants from Bruker, outside the submitted work. M.C. Fisher reports grants and personal fees for lectures from Gilead Sciences Ltd, outside the submitted work.

Support statement: This work was supported by the Natural Environment Research Council (grant NE/P001165/1), Medical Research Council (grant MR/R015600/1) and Canadian Institute for Advanced Research. Funding information for this article has been deposited with the Crossref Funder Registry. 


\section{References}

1 Zhou F, Yu T, Du R, et al. Clinical course and risk factors for mortality of adult inpatients with COVID-19 in Wuhan, China: a retrospective cohort study. Lancet 2020; 395: 1054-1062.

2 Chen N, Zhou M, Dong X, et al. Epidemiological and clinical characteristics of 99 cases of 2019 novel coronavirus pneumonia in Wuhan, China: a descriptive study. Lancet 2020; 395: 507-513.

3 Rawson TM, Moore LSP, Zhu N, et al. Bacterial and fungal co-infection in individuals with coronavirus: a rapid review to support COVID-19 antimicrobial prescribing. Clin Infect Dis 2020: in press [https://doi.org/10.1093/cid/ ciaa530].

4 Hwang DM, Chamberlain DW, Poutanen SM, et al. Pulmonary pathology of severe acute respiratory syndrome in Toronto. Mod Pathol 2005; 18: 1-10.

5 Wang H, Ding Y, Li X, et al. Fatal aspergillosis in a patient with SARS who was treated with corticosteroids. N Engl J Med 2003; 349: 507-508.

6 Milne-Price S, Miazgowicz KL, Munster VJ. The emergence of the Middle East respiratory syndrome coronavirus. Pathog Dis 2014; 71: 121-136.

7 Yang $\mathrm{X}, \mathrm{Yu} \mathrm{Y}, \mathrm{Xu}$ J, et al. Clinical course and outcomes of critically ill patients with SARS-CoV-2 pneumonia in Wuhan, China: a single-centered, retrospective, observational study. Lancet Respir Med 2020; 8: 475-481.

8 Rutsaert L, Steinfort N, Van Hunsel T, et al. COVID-19-associated invasive pulmonary aspergillosis. Ann Intensive Care 2020; 10: 71.

9 Alanio A, Delliere S, Fodil S, et al. Prevalence of putative invasive pulmonary aspergillosis in critically ill patients with COVID-19. Lancet Respir Med 2020; 8: e48-e49.

10 van Arkel ALE, Rijpstra TA, Belderbos HNA, et al. COVID-19 associated pulmonary aspergillosis. Am J Respir Crit Care Med 2020; 202: 132-135.

11 Koehler P, Cornely OA, Bottiger BW, et al. COVID-19 associated pulmonary aspergillosis. Mycoses 2020; 63: 528-534.

12 Wauters J, Baar I, Meersseman P, et al. Invasive pulmonary aspergillosis is a frequent complication of critically ill H1N1 patients: a retrospective study. Intensive Care Med 2012; 38: 1761-1768.

13 Schauwvlieghe A, Rijnders BJA, Philips N, et al. Invasive aspergillosis in patients admitted to the intensive care unit with severe influenza: a retrospective cohort study. Lancet Respir Med 2018; 6: 782-792.

14 van de Veerdonk FL, Kolwijck E, Lestrade PP, et al. Influenza-associated aspergillosis in critically ill patients. Am J Respir Crit Care Med 2017; 196: 524-527.

15 Crum-Cianflone NF. Invasive Aspergillosis associated with severe influenza infections. Open Forum Infect Dis 2016; 3: ofw171.

16 Donnelly JP, Chen SC, Kauffman CA, et al. Revision and update of the Consensus Definitions of Invasive Fungal Disease from the European Organization for Research and Treatment of Cancer and the Mycoses Study Group Education and Research Consortium. Clin Infect Dis 2019: in press [https://doi.org/10.1093/cid/ciz1008].

17 Recovery: Randomised Evaluation of Covid-19 Therapy. 2020 Low-cost dexamethasone reduces death by up to one third in hospitalised patients with severe respiratory complications of COVID-19. www.recoverytrial.net/files/ recovery_dexamethasone_statement_160620_final.pdf Date last updated: 16 June 2020; date last accessed: 17 June 2020.

18 Lahmer T, Rasch S, Spinner C, et al. Invasive pulmonary aspergillosis in severe COVID-19 pneumonia. Clin Microbiol Infect 2020; in press [https://doi.org/10.1016/j.cmi.2020.05.032].

19 Blaize M, Mayaux J, Nabet $\mathrm{C}$, et al. Fatal invasive aspergillosis and coronavirus disease in an immunocompetent patient. Emerg Infect Dis 2020; 26: 1636-1637.

20 Prattes J, Valentin T, Hoenigl M, et al. Invasive pulmonary aspergillosis complicating COVID-19 in the ICU - a case report. Med Mycol Case Rep 2020; in press [https://doi.org/10.1016/j.mmcr.2020.05.001].

21 Lescure FX, Bouadma L, Nguyen D, et al. Clinical and virological data of the first cases of COVID-19 in Europe: a case series. Lancet Infect Dis 2020; 20: 697-706.

22 Antinori S, Rech R, Galimberti L, et al. Invasive pulmonary aspergillosis complicating SARS-CoV-2 pneumonia: a diagnostic challenge. Travel Med Infect Dis 2020; in press [https://doi.org/10.1016/j.tmaid.2020.101752].

23 Docherty AB, Harrison EM, Green CA. Features of 16,749 hospitalised UK patients with COVID-19 using the ISARIC WHO Clinical Characterisation Protocol. medRxiv 2020; in press [https://doi.org/10.1101/2020.04.23. 20076042].

24 Blot SI, Taccone FS, Van den Abeele AM, et al. A clinical algorithm to diagnose invasive pulmonary aspergillosis in critically ill patients. Am J Respir Crit Care Med 2012; 186: 56-64.

25 ISRCTN registry. Aspergillosis in patients with severe influenza or coronavirus. ISRCTN51287266. Date last updated: 9 April 2020; date last accessed: 17 July 2020.

26 van de Veerdonk FL, Bruggerman R, Wauters J, et al. Dutch-Belgian Mycosis Study Group: CAPA. [serial online] 2020. https://data.castoredc.com/.

27 GOV.UK. COVID-19 safe handling and processing for samples in laboratories. www.gov.uk/government/publications/ wuhan-novel-coronavirus-guidance-for-clinical-diagnostic-laboratories/wuhan-novel-coronavirus-handling-and-processingof-laboratory-specimens Date last updated: 16 July 2020; date last accessed: 17 July 2020.

28 Heldt S, Hoenigl M. Lateral flow assays for the diagnosis of invasive aspergillosis: current status. Curr Fungal Infect Rep 2017; 11: 45-51.

29 Barnes RA, White PL, Morton CO, et al. Diagnosis of aspergillosis by PCR: clinical considerations and technical tips. Med Mycol 2018; 56: 60-72.

30 Li Y, Xia L. Coronavirus disease 2019 (COVID-19): role of chest CT in diagnosis and management. AJR Am J Roentgenol 2020: 214: 1280-1286.

31 Meersseman W, Lagrou K, Maertens J, et al. Galactomannan in bronchoalveolar lavage fluid: a tool for diagnosing aspergillosis in intensive care unit patients. Am J Respir Crit Care Med 2008; 177: 27-34.

32 Verweij PE, Snelders E, Kema GHJ, et al. Azole resistance in Aspergillus fumigatus: a side-effect of environmental fungicide use? Lancet Infect Dis 2009; 9: 789-795.

33 Lahmer T, Neuenhahn M, Held J, et al. Comparison of 1,3-beta-d-glucan with galactomannan in serum and bronchoalveolar fluid for the detection of aspergillus species in immunosuppressed mechanical ventilated critically ill patients. J Crit Care 2016; 36: 259-264. 
34 De Vlieger G, Lagrou K, Maertens J, et al. Beta-D-glucan detection as a diagnostic test for invasive aspergillosis in immunocompromised critically ill patients with symptoms of respiratory infection: an autopsy-based study. J Clin Microbiol 2011; 49: 3783-3787.

35 Wahidi MM, Shojaee S, Lamb CR, et al. The use of bronchoscopy during the COVID-19 pandemic: CHEST/ AABIP Guideline and Expert Panel Report. Chest 2020; in press [https://doi.org/10.1016/j.chest.2020.04.036].

36 Patterson TF, Thompson GR, 3rd, Denning DW, et al. Practice guidelines for the diagnosis and management of aspergillosis: 2016 update by the Infectious Diseases Society of America. Clin Infect Dis 2016; 63: e1-e60.

37 Ullmann AJ, Aguado JM, Arikan-Akdagli S, et al. Diagnosis and management of Aspergillus diseases: executive summary of the 2017 ESCMID-ECMM-ERS guideline. Clin Microbiol Infect 2018; 24: Suppl. 1, el-e38. 